\title{
Advances in cardiac PET/MR imaging: Facilitating cutting-edge structural and biological phenotyping of the cardiovascular system
}

\author{
Ahmed Tawakol, MD, ${ }^{\mathrm{a}, \mathrm{b}, \mathrm{g}}$ Georges El Fakhri, PhD, ${ }^{\mathrm{c}, \mathrm{d}, \mathrm{g}}$ Ciprian Catana, MD, \\ PhD, e,g and David E. Sosnovik, MD ${ }^{a, e, f, g}$ \\ a Cardiology Division, Massachusetts General Hospital, Boston, MA \\ b Cardiovascular Imaging Research Center, Massachusetts General Hospital, Boston \\ c Gordon Center for Medical Imaging, Massachusetts General Hospital, Boston \\ d Nuclear Medicine\& Molecular Imaging Division, Massachusetts General Hospital, Boston \\ e Athinoula A. Martinos Center for Biomedical Imaging, Massachusetts General Hospital, Boston \\ f Cardiovascular Research Center at Massachusetts General Hospital, Boston, MA \\ g Harvard Medical School, Boston, MA
}

Received Jan 12, 2020; accepted Jan 13, 2020

doi: 10.1007/s12350-020-02076-6

\section{See related article, pp. 2014-2025}

The past decade has seen a dramatic increase in the clinical adoption of cardiac positron emission tomography (PET). Integrated PET and computed tomography (PET-CT) is now routinely used to assess myocardial perfusion and to evaluate for myocardial viability, cardiac sarcoidosis and infections. In the next decade, we believe that the clinical utility of cardiac PET imaging will continue to grow, facilitated by innovations in two key areas: (1) the development of novel targeted tracers of several molecular pathways, and (2) improvements in imaging hardware and reconstruction techniques. Among developments in instrumentation, integrated PET and magnetic resonance imaging (PET-MRI) scanners represents one of the most exciting opportunities.

Integrated PET-MRI scanners were initially developed in the late 1990s and were first commercialized as a clinical imaging instrument in 2010. Since then, dozens of PET-MRI scanners have been installed across the

\footnotetext{
Reprint requests: Ahmed Tawakol, MD, Cardiology Division, Massachusetts General Hospital, Yaw 5-050, 55 Fruit Street, Boston, MA 02114; atawakol@mgh.harvard.edu

J Nucl Cardiol 2021;28:2026-9.

$1071-3581 / \$ 34.00$

Copyright (c) 2020 American Society of Nuclear Cardiology.
}

world. While much of the clinical use of PET-MRI has focused on neurological and oncological conditions, the platform also holds great promise for advanced cardiovascular imaging. At the present time, however, PETMRI remains mainly a research tool, in part due to the need to more fully solve several technical hurdles.

One challenging technical issue for cardiovascular PET-MRI is the generation of accurate attenuation correction (AC) maps. PET imaging requires correction for the attenuation effects that occur as $511 \mathrm{keV}$ annihilation photons traverse body tissues. PET-CT systems utilize data from low-dose CT scans to produce attenuation maps ( $\mu$-maps), taking advantage of the fact that linear attenuation coefficients are closely approximated from the electron density (i.e., Hounsfield units) in the CT image volume. In contrast, MR images reflect the distribution of protons, and the conversion of MR data into $\mu$-maps is thus more difficult. ${ }^{1}$ One commonly employed method to derive $\mu$-maps from MR data is to segment tissues broadly into lung, fat, soft tissue, and background using a multipoint Dixon sequence. ${ }^{2}$ However, this approach has notable weaknesses, including the inability to segment bone and calcification, which results in an underestimation of attenuation values near bone (e.g., retrosternal cardiac structures ${ }^{3}$ ) or in calcified plaques and valves. Further, the Dixon technique is substantially affected by motion artifacts (notably along the heart-lung and liver-lung interfaces). To potentially overcome these issues, a free-breathing radial Gradient Recalled Echo (GRE) approach was developed. ${ }^{4}$ The 
study by Andrews et al. provides a needed evaluation of the relative utility of the Dixon vs radial GRE approaches for cardiac PET-MR imaging.

In their study, 18 patients with aortic stenosis $(n=7)$ or recent myocardial infarction $(N=11)$ underwent ${ }^{18}$ F-fluoride PET-CT followed by PET-MRI. The PET-MRI data were reconstructed using standard (Dixon) and novel (radial GRE) MR $\mu$-maps, and both PET-MRI datasets were compared to data obtained using PET-CT. Uptake of ${ }^{18}$ F-fluoride (which accumulates in proportion to active calcification) was evaluated within the aortic valves and coronary arteries. The authors reported that aortic valve ${ }^{18} \mathrm{~F}$-fluoride uptake appeared visually similar on PET-CT and PET-MRI. Additionally, compared to PET-CT, background-corrected uptake values were comparable with radial GREbased AC but correlated less well with Dixon-based AC. The authors also report that coronary ${ }^{18} \mathrm{~F}$-fluoride uptake was similar between PET-MRI and PET-CT regardless of the AC approach. However, it was notable that coronary stents caused artifact on PET-MR, making assessment of ${ }^{18} \mathrm{~F}$-fluoride uptake challenging.

While we largely agree with the author's conclusions, and commend them for performing this excellent work, several aspects of the study merit further discussion. The authors focused their analysis on the correlation of standardized uptake values (SUV) and target-to-background ratios (TBR) by CT and MR. While these are important metrics, future expansion of this approach should include a receiver operator characteristic analysis (ROC) of the PET-MRI data using the PET-CT data as the gold standard. ROC analyses could quantify the ability of PET-MRI to accurately detect aortic and coronary calcification activity and define the optimal thresholds for each AC method. This point is well demonstrated by patients 2 and 4 in Figure 4 of the paper: In both patients, clear foci of calcification and ${ }^{18} \mathrm{~F}$-floride uptake are present in the LAD. In both cases these foci are not at all detected with the GRE radial AC method but are well detected with the Dixon AC method. Furthermore, in patient 4 , a false-positive focus of ${ }^{18} \mathrm{~F}$-fluoride is present in the aorta with the radial GRE AC method but not the Dixon approach. Likewise, in Figure 3, the detection of the posterior mitral annular calcification activity seems more robust with the radial GRE approach, but the focus of calcification in the descending aorta is missed. These brief snapshots suggest that an ROC analysis would provide important and complementary data to the correlation data.

It is important to note however, that an ROC analysis converts continuous data (SUV, TBR) into binary data (above or below threshold) and thus would not assess the ability of PET-MRI to quantify serial changes that fall above/below the detection threshold. For this purpose, the correlation of SUV/TBR values across the full spectrum is the more relevant metric. Nonetheless, we would submit that complete conclusions on the performance of PET-MR would be enhanced by the use of an ROC-derived metric of detectability or diagnostic accuracy. From the data presented, it seems clear that the Dixon AC approach does produce more extra-cardiac artifacts, but it is unclear if they would impact the diagnostic accuracy of this AC approach for the detection of aortic valve or coronary calcium. This question, as discussed above, would be fully addressed with an ROC analysis in future studies.

The SUVs in the blood pool, aortic valve and coronary arteries were all higher on PET-CT than PETMRI. However, all the subjects in the study were imaged with PET-CT first, followed by PET-MRI, suggesting that this effect was due to the kinetics of probe elimination and not the imaging technique. In a future study it would be interesting to alternate or randomize the order of imaging (i.e., whether PET-CT or PET-MRI occurs first) to more fully explore this. Regardless, the use of TBR substantially compensates for variable kinetics and is, therefore, the more relevant metric to assess in this study.

Additional development of this work should leverage methods to correct for susceptibility artifacts induced by implanted materials (e.g., stents, bioprosthetic valves, TAVR), in order to allow more complete assessments of tracer activity in patients with such materials. For example, applying a morphological closing operation or using the approach suggested by Lassen et al. ${ }^{5}$ would address this issue and enable the characterization of ${ }^{18} \mathrm{~F}$-floride uptake using PET-MRI in the stented coronaries as well. As Lassen et al. showed in several patients with metallic implants, the uptake in the affected coronary segments changed significantly after applying the artifact correction, leading to a change in the clinical assessment of those patients. It would be informative to assess the correlation between the uptake values obtained by PET-MRI after applying such a correction (even manually) and those obtained by PET/ CT.

While several questions remain unanswered, the excellent study by Andrews et al. should provide confidence to those who wish to image native aortic valves and unstented segments of the coronary arteries by PETMRI. Although further study will be needed to determine the optimal AC technique, if the study is adequately powered, both Dixon and Radial GRE will likely perform well. Simultaneously, it will also be important to better understand the failure modes of both techniques and in which scenarios one technique might outperform another. Intuitively, it would seem surprising 
for a 4-parameter model (Dixon) to be outperformed by a 2-parameter model (Radial GRE).

Although the optimal algorithm for AC remains a relative challenge for PET-MRI, the potential for major advances is excellent in other technical domains. One such area is in the use the simultaneously derived MR data (which provides data on tissue motion with excellent tissue contrast, spatial and temporal resolution) to more accurately correct the 3D motion of the PET dataset. ${ }^{6}$ Further, MR data can be leveraged for correction of partial volume effects in order to improve PET quantitation. ${ }^{7}$ This approach may be less susceptible to misregistration seen when structural imaging is not simultaneously derived with PET (as is the case with PET-CT). Together, the development of each of these techniques is enhancing the utility of PET-MRI as a quantitative PET imaging tool.

However, the greatest advantages of PET-MRI over PET-CT lie not solely in the opportunity to use it to improve PET data quantification. One notable, additional advantage of PET/MRI relative to PET-CT is the reduced radiation exposure enabled by PET/MRI. Moreover, perhaps PET/MRI's greatest advantage lies in the ability to simultaneously derive complementary PET and MRI assessments of the cardiovascular system. MRI has become the gold standard for characterization of left and right ventricular function and of myocardial tissue (including scar, edema, and fat), and provides excellent assessments of viability and vessel wall and valvular pathologies. ${ }^{8}$ On the other hand, PET provides accurate assessments of myocardial perfusion and provides noninvasive assessments of molecular processes, including metabolism, inflammation, innervation, and active calcification. ${ }^{9}$ Hence the combination of PET and MR data has the potential to provide a particularly robust structural and biological phenotyping of the cardiovascular system.

The optimal clinical use of cardiovascular PETMRI remains unclear. Most current clinical applications of cardiovascular PET-MRI use protocols that were developed for either PET or MRI in isolation. However, it is imperative that dedicated protocols are developed to combine the PET and MRI datasets in a manner that yields a unique multi-modality assessment. Ideally, such PET/MRI assessments should provide synergistic information that is more clinically useful than if the PET and MR imaging were simply performed on separate scanners. Furthermore, such protocols will eventually need to prove to be cost-effective. To that end, studies have begun to evaluate the synergistic value of PET and MRI data obtained using PET-MRI. In one study, Dweck et al. ${ }^{10}$ evaluated for active cardiac sarcoidosis using both PET and MRI data derived simultaneously on PET-MRI: (1) though identifying high myocardial FDG uptake $(\mathrm{PET}+)$ and (2) though identifying late gadolinium enhancement $(\mathrm{MRI}+)$. In their pilot study, they posited that the combined PET and MRI data improved identification of sarcoidosis, compared to use of either modality on its own. Specifically, individuals with positive signals coming from both modalities (PET and MRI double-positives) represented true active sarcoid, while cases that yielded only single-positives represented either false-positives or inactive disease. While more research is needed to validate and extend these observations, the combined use of PET-MRI for sarcoidosis imaging shows potential to yield a more accurate assessment of the disease.

Other areas of development in cardiac PET-MR imaging include multi-modality evaluation of atherosclerotic plaques, vasculitis, inflammation after myocardial infarction, cardiomyopathies, and others. Additionally, PET-MR imaging can be leveraged to evaluate disease mechanisms that span several tissues. For example, PET-MR imaging was used to identify a multi-tissue pathway linking chronic stress to cardiovascular disease events, involving: $\uparrow$ stress-associated neurobiological activity, $\uparrow$ bone marrow leukopoiesis, $\uparrow$ arterial inflammation and $\uparrow$ non-calcified coronary plaques. ${ }^{11,12}$ Thus, advanced multi-modality, multi-organ imaging has the potential to uncover complex disease mechanism, where single-system imaging might leave them hidden.

Despite PET-MRI's substantial promise as a cardiovascular imaging tool, much work needs yet to be done before its use becomes more widespread. Continued progress will require close collaboration among individuals trained in cardiac PET, cardiac MRI, and medical physics. Advances will be further accelerated as new trainees with specialized training in both cardiac PET and cardiac MRI join such teams. Together, such efforts will enhance the utility of cardiovascular PETMR imaging, enabling powerful structural and biological phenotyping of the cardiovascular system, and ultimately improving care.

\section{Disclosure}

AT received minor consulting fees (from Actelion Inc and Esperion) and grants to his institution (from Genentech), for work that is unrelated to that contained in the manuscript.

\section{References}

1. Hofmann M, Pichler B, Scholkopf B, Beyer T. Towards quantitative PET/MRI: A review of MR-based attenuation correction techniques. Eur J Nucl Med Mol Imaging 2009;36:S93-S104.

2. Martinez-Möller A, Souvatzoglou M, Delso G, Bundschuh RA, Chefd'hotel C, Ziegler SI, Navab N, Schwaiger M, Nekolla SG. 
Tissue classification as a potential approach for attenuation correction in whole-body PET/MRI: Evaluation with PET/CT data. J Nucl Med 2009;50:520-6.

3. Samarin A, Burger C, Wollenweber SD, Crook DW, Burger IA, Schmid DT, von Schulthess GK, Kuhn FP. PET/MR imaging of bone lesions-implications for PET quantification from imperfect attenuation correction. Eur $\mathrm{J}$ Nucl Med Mol Imaging 2012;39:1154-60.

4. Robson PM, Dweck MR, Trivieri MG, Abgral R, Karakatsanis NA, Contreras J, Gidwani U, Narula JP, Fuster V, Kovacic JC, Fayad ZA. Coronary artery PET/MR imaging: Feasibility, limitations, and solutions. JACC Cardiovasc Imaging 2017;10:110312.

5. Lassen ML, Rasul S, Beitzke D, Stelzmüller M-E, Cal-Gonzalez J, Hacker M, Beyer T. Assessment of attenuation correction for myocardial PET imaging using combined PET/MRI. J Nucl Cardiol 2017;26:1107-18.

6. Petibon Y, Ouyang J, Zhu X, Huang C, Reese TG, Chun SY, Li Q, Fakhri GE. Cardiac motion compensation and resolution modeling in simultaneous PET-MR: A cardiac lesion detection study. Phys Med Biol 2013;58:2085-102.

7. Chen KT, Salcedo S, Gong K, Chonde DB, Izquierdo-Garcia D, Drzezga AE, Rosen B, Qi J, Dickerson BC, Catana C. An efficient approach to perform MR-assisted PET data optimization in simultaneous PET/MR neuroimaging studies. J Nucl Med 2018;60:272-8.

8. American College of Cardiology Foundation Task Force on Expert Consensus D, Hundley WG, Bluemke DA, Finn JP, Flamm SD, Fogel MA, Friedrich MG, Ho VB, Jerosch-Herold M, Kramer CM, Manning WJ, Patel M, Pohost GM, Stillman AE, White RD, Woodard PK. ACCF/ACR/AHA/NASCI/SCMR 2010 expert consensus document on cardiovascular magnetic resonance: A report of the American College of Cardiology Foundation Task
Force on Expert Consensus Documents. J Am Coll Cardiol 2010;55:2614-62.

9. Dilsizian V, Bacharach SL, Beanlands RS, Bergmann SR, Delbeke D, Dorbala S, Gropler RJ, Knuuti J, Schelbert HR, Travin MI. ASNC imaging guidelines/SNMMI procedure standard for positron emission tomography (PET) nuclear cardiology procedures. J Nucl Cardiol 2016;23:1187-226.

10. Dweck MR, Abgral R, Trivieri MG, Robson PM, Karakatsanis N, Mani V, Palmisano A, Miller MA, Lala A, Chang HL, Sanz J, Contreras J, Narula J, Fuster V, Padilla M, Fayad ZA, Kovacic JC. Hybrid magnetic resonance imaging and positron emission tomography with fluorodeoxyglucose to diagnose active cardiac sarcoidosis. JACC Cardiovasc Imaging 2018;11:94-107.

11. Tawakol A, Ishai A, Takx RA, Figueroa AL, Ali A, Kaiser Y, Truong QA, Solomon CJ, Calcagno C, Mani V, Tang CY, Mulder WJ, Murrough JW, Hoffmann U, Nahrendorf M, Shin LM, Fayad ZA, Pitman RK. Relation between resting amygdalar activity and cardiovascular events: A longitudinal and cohort study. Lancet 2017;389:834-45.

12. Goyal A, Dey AK, Chaturvedi A, Elnabawi YA, Aberra TM, Chung JH, Belur AD, Groenendyk JW, Lerman JB, Rivers JP, Rodante JA, Harrington CL, Varghese NJ, Sanda GE, Baumer Y, Sorokin AV, Teague HL, Genovese LD, Natarajan B, Joshi AA, Playford MP, Bluemke DA, Chen MY, Alavi A, Pitman RK, Powell-Wiley TM, Tawakol A, Gelfand JM, Mehta NN. Chronic stress-related neural activity associates with subclinical cardiovascular disease in psoriasis: A prospective cohort study. JACC 2018;13:465-77.

Publisher's Note Springer Nature remains neutral with regard to jurisdictional claims in published maps and institutional affiliations. 